\title{
On the NBC-Bases of product hypersolvable arrangements
}

\author{
Mayadah Abd Ulrazaq \\ University of Basrah, College of Science, Department of Mathematics, \\ Basrah, Iraq \\ Email: mayadah.almolany@yahoo.com
}

Recived : 17>7/2018

Revised : 261712018

Accepted : 11812018

Available online : $\quad 6 / 8 / 2018$

DOI: $10.29304 / j q c m .2018 .10 .3 .411$

\begin{abstract}
This paper aims centered around the product of hypersolvable arrangements by using the hypersolvable partition analogue by proving that each of $A$ and $B$ are hypersolvable if $A \times B$ is a hypersolvable, also each of $A$ and $B$ are supersolvable if $A \times B$ is supersolvable. Moreover, this paper show how to prove that the dimension of the first non-vanishing higher homotopy groups of the complement $M(A \times B)$ is $p(A \times B)=\min \{p(A), p(B)\}$.
\end{abstract}

Keyword: Hypersolvable, Supersolvable, Hypersolvable arrangements

List of symbols: $r k(A)=\operatorname{rank}$ of $A, T(A)=$ maximal element of central $A, M(A)=$ complement of $A$.

Mathematics subject classification: 55Q20 
Mayadah .A

\section{Introduction:}

Let $A=\left\{H_{1}, \ldots, H_{n}\right\}$ be a complex hyperplane $r$-arrangement, with complement $M(A)=C^{r} \backslash \bigcup_{i=1}^{n} H_{i}$. The cohomology ring for the complement $M(A)$, with arbitrary constant coefficients was given by Arnold [1] and Brieskorn [2].

For a given total order $\unlhd$ on $A$, if $C \subseteq A$ is a minimal (with respect to inclusion) dependent set, we call $C$ a circuit of $A$ and $\bar{C}=C \backslash\{H\}$ a broken circuit of $C$, where $H$ is the smallest hyperplane in $C$ via $\unlhd$ and by NBC base $B \subseteq A$ we mean that $B$ contains no broken circuit.

The hypersolvable class of hyperplane arrangements were originally introduced by $\mathrm{M}$. Jambu and S. Papadima [3,4], as a combinatorial generalization of the supersolvable class of hyperplane arrangements and they showed that all the major results on the topology of the complements together with their algebraic and combinatorial aspects, may be extended and refined in this new framework. The hypersolvable class of hyperplane arrangements contains the supersolvable ones, the generic ones and many others.

We used the hypersolvable partition, the hypersolvable ordering which are defined by Ali and Al-Ta'ai [5], and their study of the NBC bases of a hypersolvable arrangement to complete the study of the product of hypersolvable arrangements which is studied by Mahdi in [ 7 ] he suggested a conjecture , namely, if $(A \times B, V \oplus W) \quad$ is a hypersolvable arrangement, then $A$ and $B$ are hypersolvable arrangements. This conjecture is proved under some condition, namely, all the exponents of $A \times B$ are equal to 1 . In section three we prove this conjecture without any condition, also we prove that the dimension of the first non-vanishing higher homotopy groups for complement $M(A \times B)$ is $p(A \times B)=\min \{p(A), p(B)\}$.

\section{A hypersolvable partition of an arrangement}

A hypersolvable class of arrangements was originally introduced by Jambu and Papadima ([3], [4]) Ali and Al-Ta'ai redefine this concept by using a partition which is called a hypersolvable partition as follows:

\section{(2.1)Definition: [5]}

Let $A$ be an essential central complex $r$ arrangement(i.e. $\bigcap_{i=1}^{n} H_{i}=T(A)=T \neq \phi$ and $\operatorname{rk}(A)=\operatorname{rk}(T(A))=\operatorname{codim}\left(\bigcap_{H \in A} H=r=\right.$ $\left.\operatorname{dim}\left(C^{r}\right)\right)$. A partition $\Pi=\left(\Pi_{1}, \ldots, \Pi_{\ell}\right)$ of $A$ is said to be a hypersolvable partition of $A$ with length $\ell(A)=\ell$ denoted by $H p$, if $\left|\Pi_{1}\right|=1$, (i.e. $\Pi_{1}$ is a singleton), and for fixed $2 \leq j \leq \ell$, the block $\Pi_{j}$ satisfies the following properties:

( j-closed property of $\Pi_{j}$ ) For each $H_{1}, H_{2} \in \Pi_{1} \cup \cdots \cup \Pi_{j}$, there is no hyperplane $\quad H \in \Pi_{j+1} \cup \cdots \cup \Pi_{\ell}$ such
$\operatorname{that} \operatorname{rk}\left(H_{1}, H_{2}, H\right)=2$. 
( j-complete property of $\Pi_{j}$ ) For each $H_{1}, H_{2} \in \Pi_{j}$, there is a hyperplane $H \in \Pi_{1} \cup \cdots \cup \Pi_{j-1} \quad$ such $\quad$ that $\operatorname{rk}\left(H_{1}, H_{2}, H\right)=2$. Note that, from the closed properties of the blocks $\Pi_{2}, \ldots, \Pi_{j-1}$, the hyperplane $H$ is unique and it is denoted in this case by $H_{1,2}$.

( $j$-solvable property of $\left.\quad \Pi_{j}\right) \quad$ If $H_{1}, H_{2}, H_{3} \in \Pi_{j}$, the hyperplanes $H_{1,2}, H_{1,3}, H_{2,3} \in \Pi_{1} \cup \cdots \cup \Pi_{j-1} \quad$ are equal $\operatorname{or} \operatorname{rk}\left(H_{1,2}, H_{1,3}, H_{2,3}\right)=2$. Observe that, if $\operatorname{rk}\left(H_{1}, H_{2}, H_{3}\right)=2$, then from the closed properties of the blocks $\Pi_{2}, \ldots, \Pi_{j-1}$, we have $H_{1,2}=H_{1,3}=H_{2,3}$.

The vector of integers $d=\left(d_{1}, \ldots, d_{\ell}\right)$, is called the exponent vector of $\Pi$, where $d_{i}=\left|\Pi_{i}\right|$, $i=1, \ldots, \ell$. The rank of $\Pi_{i}$ is defined to be $\operatorname{rk}\left(\Pi_{i}\right)=\operatorname{rk}\left(\Pi_{1} \cup \cdots \cup \Pi_{i}\right)=\operatorname{rk}\left(\bigcap_{H \in \Pi_{1} \cup \cdots \cup \Pi_{i}} H\right)$ , for $1 \leq i \leq \ell$. We call the block $\Pi_{i}$, a singular block of $\Pi$ if $\operatorname{rk}\left(\Pi_{i}\right)=\operatorname{rk}\left(\Pi_{i-1}\right)$ and we call it non-singular block otherwise. Notice that, in general $\operatorname{rk}\left(\Pi_{i}\right) \leq \operatorname{rk}\left(\Pi_{i-1}\right)+1$

\section{(2.2) Proposition: [6]}

Let $A$ be an essential central complex $r$-arrangement. $A$ is hypersolvable if, and only if, $A$ has a Hp $\prod=\left(\prod_{1}, \ldots, \prod_{\ell}\right)$.

\section{(2.3) Definition:[5]}

Let $A$ be a hypersolvable $r-$ arrangement with $\mathrm{Hp} \Pi=\left(\prod_{1}, \ldots, \prod_{\ell}\right)$. For a fixed $1 \leq j \leq \ell$, the properties of the hypersolvable partition give rise to a natural partition $\prod_{j}$ as follows:

1- Let $\prod_{j * 1}=\left\{H_{i_{1}}, \ldots, H_{i_{k}}\right\}$ such that $r k\left(H_{i_{1}}, \ldots, H_{i_{k}}\right)=2$ and 2- Let $\prod_{j * 2}=\prod_{j} \backslash \prod_{j * 1}$.

Define the hypersolvable ordering of $A$ that is denoted by $\unlhd$ as follows:

1- $\quad H \in \prod_{i}$ and $H^{\prime} \in \prod_{j} \quad$ such that $1 \leq i<j \leq \ell$, put $H \unlhd H^{\prime}$.

2- For a fixed $1<j \leq \ell$, give the hyperplanes of the block $\prod_{j * 1}$ of $\prod_{j}$ an arbitrary total order with preserving the order of $\prod_{i}$ in $\Pi$ for each $1 \leq i \leq j-1$ and preserving the order of $\prod_{j * 2}$ as $\quad$ if $\quad H_{1}, H_{2}, H_{3} \in \prod_{j} \quad$ with $r k\left(H_{1}, H_{2}, H_{3}\right)=3$, put $H_{i_{1}} \unlhd H_{i_{2}} \unlhd H_{i_{3}}$ if, and only if, $H_{i_{1}, i_{2}} \triangleleft H_{i_{2}, i_{3}} \unlhd H_{i_{1}, i_{3}}$ such that $\left\{H_{i_{1}}, H_{i_{2}}, H_{i_{3}}\right\}=\left\{H_{1}, H_{2}, H_{3}\right\} . \quad$ Observe that, since $r k\left(H_{1}, H_{2}, H_{3}\right)=3$ then there is at least one of $H_{1}, H_{2}, H_{3} \in \prod_{j *_{2}}$.

\section{(2.4) proposition:[3]}

Let $A$ be a hypersolvable arrangement. Then $A$ is said to be supersolvable if, and only if, $\ell(A)=r k(A)$. 
Mayadah .A

\section{The Product of Hypersolvable}

\section{Arrangement}

\section{(3.1) Definition:}

Let $(A, V)$ and $(B, W)$ be two hyperplane arrangements. Define the product $(A \times B, V \oplus W) \quad$ by $A \times B=\{H \oplus W: H \in A\} \bigcup\{V \oplus K: K \in B\}$

Note that, $|A \times B|=|A|+|B|$. If we denote the sets $\{H \oplus W: H \in A\}$ and $\{V \oplus K: K \in B\}$ by $A \oplus W$ and $V \oplus B$ respectively, then one can easily denote the hyperplane arrangement $A \times B$ by $A \times B=(A \oplus W) \cup(V \oplus B)$.

\section{(3.2) Proposition: [7]}

Let $(A \times B, V \oplus W)$ be the product of $(A, V)$ and $(B, W)$ such that, $r k(A)=r$ and $r k(B)=k$. Then we have the following:

1. If each one of $A$ and $B$ is a hypersolvable arrangement, then $(A \oplus W, V \oplus W)$,

$(V \oplus B, V \oplus W)$ and

$(A \times B, V \oplus W)$ are hypersolvable arrangements.

2. If each one of $A$ and $B$ is a supersolvable arrangement, then $(A \oplus W, V \oplus W)$, $(V \oplus B, V \oplus W)$ and $(A \times B, V \oplus W) \quad$ are supersolvable arrangements .

\section{(3.3) Remark:}

Suppose $(A, V)$ and $(B, W)$ be hypersolvable arrangements with hypersolvable partitions say; $\prod^{A}=\left(\prod_{1}^{A}, \ldots, \prod_{\ell_{1}}^{A}\right)$ and $\Pi^{B}=\left(\prod_{1}^{B}, \ldots, \prod_{\ell_{2}}^{B}\right)$ respectively. From [7], then $(A \times B, V \oplus W) \quad$ is a hypersolvable arrangement with a hypersolvable composition series ;

$\prod_{1}^{A} \oplus W \subseteq\left(\prod_{1}^{A} \cup \prod_{2}^{A}\right) \oplus W \subseteq \ldots$

$\subseteq\left(\prod_{1}^{A} \cup \ldots \cup \prod_{\ell_{1}}^{A}\right) \oplus W=(A \oplus W)$

$\subseteq(A \oplus W) \bigcup V \oplus \prod_{1}^{B} \subseteq \quad$ From

$\ldots \subseteq(A \oplus W) \cup V \oplus\left(\prod_{1}^{B} \cup \ldots \cup \prod_{\ell_{2}}^{B}\right)$

$=(A \oplus W) \bigcup(V \oplus B) \ldots . .(3.1)$

[5], $A \times B$ has a hypersolvable partition $\Pi^{A \times B}=\left(\Pi_{1}^{A \times B}, \ldots, \Pi_{\ell_{1}+\ell_{2}}^{A \times B}\right)$ induced from the composition series (3.1), as follows:

- For $1 \leq k \leq \ell_{1} ; \prod_{k}^{A \times B}=\prod_{k}^{A} \oplus W$ and;

$\ell_{1}+1 \leq k \leq \ell_{1}+\ell_{2} ; \prod_{k}^{A \times B}=V \oplus \prod_{k-\ell_{1}}^{B}$.

Ali in [5] showed that such partition forms a hypersolvable partition.

\section{(3.4)Remark:[7]}

There are no collinear relations among the hyperplanes of $A \oplus W$ and $V \oplus B$. Thus, for each $H_{1}, H_{2} \in A$, there is no hyperplane $K \in B \quad$ such that $r k\left\{H_{1} \oplus W, H_{2} \oplus W, V \oplus K\right\}=2 \quad$ and for each $K_{1}, K_{2} \in B$, there is no $H \in A$ such that $r k\left\{H \oplus W, V \oplus K_{1}, V \oplus K_{2}\right\}=2$. 


\section{(3.5) Lemma:}

Every broken circuit $C$ in $A \oplus W$ has the following property; there is no hyperplane $K$ in $B$ such that $C \bigcup\{V \oplus K\}$ forms a circuit in $A \times B$. As well as, for any broken circuit $C^{\prime}$ in $V \oplus B$, there is no hyperplane $H$ in $A$ such that $C^{\prime} \bigcup\{H \oplus W\}$ forms a circuit in $A \times B$. Thus,

\section{$N B C(A \oplus W) \bigcap N B C(V \oplus B)=\phi$.}

Proof: directly result of proposition (2.4) and remark (3.3).

\section{(3.6) Proposition :}

Let $A \times B$ be a hypersolvable $r+k-$ arrangement. Then;

$$
\begin{aligned}
& N B C(A \oplus W) \subseteq N B C(A \times B) \text { and } \\
& N B C(V \oplus B) \subseteq N B C(A \times B) .
\end{aligned}
$$

Proof: By contrary, for $1 \leq k \leq r$, let $S_{k}=\left\{H_{i_{1}} \oplus W, \ldots, H_{i_{k}} \oplus W\right\} \quad$ be a $k-$ section of $\Pi^{A \times B}$, such that $S_{k} \in N B C(A \oplus W)$ and

$S_{k} \notin N B C(A \times B)$. Then $S_{k}$ be a broken circuit in $A \times B$. That is, there exists a hyperplane $H^{\prime} \in A \times B \quad$ such that $H^{\prime} \unrhd H_{i_{j}} \oplus W, 1 \leq j \leq k$ and $\left\{H^{\prime}\right\} \bigcup S_{k}$ form a circuit, i.e. $r k\left\{H^{\prime} \cup S_{k}\right\}=k$. It is clear that, $\quad H^{\prime} \notin A \oplus W, \quad$ since $S_{k} \in N B C(A \oplus W)$. On the other hand, $H^{\prime} \notin V \oplus B$ as shown in lemma (3.5) above. Therefore, $S_{k}$ must be an $N B C$ base of $A \times B$.
Similarly, it is easy to show that $N B C(V \oplus B) \subseteq N B C(A \times B)$.

\section{(3.7) Theorem:}

Let $A \times B$ be a hypersolvable $r+k-$ arrangement then;

$N B C(A \times B)=\left\{C \in A \times B \mid C=C_{1} \cup C_{2}\right.$ $: C_{1} \in N B C(A \oplus W)$ and $\left.C_{2} \in N B C(V \oplus B)\right\}$ Proof: By contrary, suppose that $C \in N B C(A \times B)$, such that $C$ cannot be written as a union of an $N B C$ base of $A \oplus W$ and $N B C$ base of $V \oplus B$, i.e. either;

$C \bigcap(A \oplus W) \notin N B C(A \oplus W)$ or $C \bigcap(V \oplus B) \notin N B C(V \oplus B)$. If $C \bigcap(A \oplus W) \notin N B C(A \oplus W)$, then there exists a hyperplane $H^{\prime} \in A \times B$ such that $H^{\prime} \cup\{C \cap(A \oplus W)\}$ forms a circuit in $A \times B$. But this contradicts our assumption that $C \in N B C(A \times B)$. By the same way, we deduce

that

$$
C \cap(V \oplus B) \notin N B C(V \oplus B) .
$$

\section{(3.8) Corollary :}

Let $A \times B$ be a hypersolvable $r+k-$ arrangement then $p(A)=p(A \oplus W)$ and $p(B)=p(V \oplus B)$.

\section{(3.9) Theorem :}

Let $A \times B$ be a hypersolvable $r+k-$ arrangement then $p(A \times B)=\min \{p(A), p(B)\}$.

Proof: In general, deduce that $p(A \times B) \leq p(A) \quad$ and $p(A \times B) \leq p(B)$. So by contrary suppose 
that, $\quad p(A \times B)<\min \{p(A), p(B)\}$. So suppose that, there exists a section $S \in S_{p(A \times B)+1}$ such that $S$ is a $(p(A \times B)+1)$-broken circuit and from our construction of $\prod^{A \times B}$ then $S=S^{A \oplus W} \bigcup S^{V \oplus B}$ where

$S^{A \oplus W}=S \bigcap A \oplus W \quad$ and $S^{V \oplus B}=S \bigcap V \oplus B . \quad$ It $\quad$ is clear that $S^{A \oplus W} \in N B C(A \oplus W)$ and

$S^{V \oplus B} \in N B C(V \oplus B)$ since $p(A \times B)+1<\min \{p(A)+1, p(B)+1\}$.

Now, let $H$ be the minimal hyperplane of $A \times B$ such that $\{H\} \bigcup S$ forms a $(p(A \times B)+1)$ circuit. If $S^{A \oplus W} \neq \phi$, then $H$ minimal than $H^{\prime}$ via the hypersolvable ordering $\unrhd$ on the hyperplanes of $A \times B$, for each $H^{\prime} \in S^{A \oplus W}$. Thus, $\quad\{H\} \bigcup S^{A \oplus W}$ is a circuit and this contradicts the fact that $S^{A \oplus W}$ is an $N B C$ base of $A \oplus W$. On the other hand, if $S^{A \oplus W}=\phi$ then $S=S^{V \oplus B}$. That is, the hyperplane $H$ minimal than $K$ via hypesovable ordering $\unrhd$ for each $K \in S^{V \oplus B}$, thus $\{H\} \bigcup S^{V \oplus B}$ is a circuit which contradicts that $S^{V \oplus B} \in N B C(V \oplus B)$. This ends the proof.

\section{(3.10)Theorem:}

If $A \times B$ be a hypersolvable $r+k-$ arrangement, then each of $A \oplus W$ and $V \oplus B$ are hypersolvable.
Proof: Since $A \times B$ be a hypersolvable $r+k$-arrangement, hence $A \times B$ has an Hp, $\prod^{A \times B}=\left(\prod_{1}, \ldots, \prod_{\ell}\right)$. From lemma (3.4), the partition $\Pi^{A \times B}$ splits into two partitions as follows:

- Let $\prod_{i}^{A}=\prod_{j_{i}}^{A \times B} \subseteq A \oplus W$, for $1 \leq i \leq \ell_{1}, 1 \leq j_{1}<j_{2}<\ldots<j_{\ell_{1}} \leq \ell$ and;

- $\prod_{i}^{B}=\prod_{j_{i}}^{A \times B} \subseteq V \oplus B \quad, \quad$ for $1 \leq i \leq \ell_{2}, 1 \leq j_{1}<j_{2}<\ldots<j_{\ell_{2}} \leq \ell ;$ where $\ell_{1}+\ell_{2}=\ell$.

Deduce that $\prod^{A}=\left(\prod_{1}^{A}, \ldots, \prod_{\ell_{1}}^{A}\right)$ form a partition of $A \oplus W$. We need to show that $\prod^{A}$ is a hypersolvable partition as follows:

1. If $\prod_{1}^{A}$ contains two hyperplanes say $H_{1} \oplus W$ and $H_{2} \oplus W$, then there exists a hyperplane $H \in \prod_{1}^{A \times B} \cup \ldots \cup \prod_{j_{n-1}}^{A \times B}$ such that $r k\left\{H_{1} \oplus W, H_{2} \oplus W, H\right\}=2$, from the complete property of block $\prod_{j_{1}}^{A \times B}$. Therefore, $H \in A \oplus W$, see lemma(3.4). But this contradicts our assumption that $\prod_{j_{1}}^{A \times B}$ is the first block of $\prod^{A \times B}$ such that $\prod_{j_{1}}^{A \times B} \subseteq A \oplus W$. Thus, $\left|\prod_{1}^{A}\right|=1$.

2. For $2 \leq k \leq \ell_{1}$; it is clear that the block $\prod_{k}^{A}$ satisfies the closed, complete and solvable properties since it is a block from an Hp. Thus $A \oplus W$ is hypersolvable since it is has an Hp. In the same way $V \oplus B$ is a hypersolvable. 


\section{(3.11) Corollary :}

The product $r+k$-arrangement $A \times B$ is hypersolvable if, and only if, each of $A$ and $B$ are hypersolvable.

Proof: It is known that, if $A$ and $B$ are hypersolvable, then $A \times B$ is hypersolvable (see [7]). Conversely, If $A \times B$ is a hypersolvable arrangement, the canonical projections $q_{A}: V \times W \rightarrow V$ defined $q_{A}(H \oplus W)=H$ and $q_{B}: W \times V \rightarrow V$ defined by $q_{B}(V \oplus K)=K$ preserve the dependent and independent relations. Therefore, each one of $A$ and $B$ are hypersolvable arrangements.

\section{(3.12) Corollary:}

$A \times B$ is supersolvable if, and only if, each of $A$ and $B$ is supersolvable.

Proof: It is known that, if $A$ and $B$ are supersolvale, then $A \times B$ is supersolvable (see [7]). Conversely if $A \times B$ is supersolvable then $\ell(A \times B)=\ell=r+k$ where $r=r k(A)$ and $k=r k(B)$, since $\ell_{1} \geq r k(A)=r$ and $\ell_{2} \geq r k(B)=k$, then $\ell=\ell_{1}+\ell_{2} \geq r+k$, but $\ell=r+k$ which means $\ell_{1}$ and $\ell_{2}$ cannot be greater than $r$ and $k$ respectively. Hence, each of $A$ and $B$ is supersolvable.

\section{(3.13) Example:}

Let $A$ be central complex 6-arrangements, define as follows:

$$
\begin{aligned}
& Q(A)=x_{2} x_{3}\left(x_{1}-x_{3}\right)\left(x_{1}+x_{3}\right)\left(x_{2}-x_{1}\right) \\
& \left(x_{2}+x_{1}\right)\left(x_{5}+3 x_{6}\right)\left(x_{5}+2 x_{6}\right) x_{5}\left(x_{5}-x_{6}\right) \\
& x_{6}\left(x_{4}+x_{5}+x_{6}\right)\left(x_{5}-x_{4}+x_{6}\right)
\end{aligned}
$$

$A$ is a hypersolvable arrangement in $C^{6}$ since we can find a hypersolvable Hp as follows:

$$
\begin{aligned}
& \Pi^{A}=\left(\Pi_{1}, \Pi_{2}, \Pi_{3}, \Pi_{4}, \Pi_{5}, \Pi_{6}, \Pi_{7}, \Pi_{8}\right) \\
& \left(\left\{H_{1}\right\},\left\{H_{2}, H_{3}\right\},\left\{H_{4}\right\},\left\{H_{5} H_{6}\right\},\left\{H_{7}\right\},\left\{H_{8}\right\},\right. \\
& \left.\left\{H_{9}\right\},\left\{H_{10}\right\},\left\{H_{11}, H_{12}, H_{13}\right\}\right) \text { where } \\
& H_{1}=\left\{\left(x_{1}, x_{2}, x_{3}, x_{4}, x_{5}, x_{6}\right): x_{1}+x_{3}=0\right\} \\
& H_{2}=\left\{\left(x_{1}, x_{2}, x_{3}, x_{4}, x_{5}, x_{6}\right): x_{1}-x_{3}=0\right\} \\
& H_{3}=\left\{\left(x_{1}, x_{2}, x_{3}, x_{4}, x_{5}, x_{6}\right): x_{1}+x_{2}=0\right\} \\
& H_{4}=\left\{\left(x_{1}, x_{2}, x_{3}, x_{4}, x_{5}, x_{6}\right): x_{2}=0\right\} \\
& H_{5}=\left\{\left(x_{1}, x_{2}, x_{3}, x_{4}, x_{5}, x_{6}\right): x_{2}-x_{1}=0\right\} \\
& H_{6}=\left\{\left(x_{1}, x_{2}, x_{3}, x_{4}, x_{5}, x_{6}\right): x_{3}=0\right\} \\
& H_{7}=\left\{\left(x_{1}, x_{2}, x_{3}, x_{4}, x_{5}, x_{6}\right): x_{5}+3 x_{6}=0\right\}
\end{aligned}
$$$$
H_{8}=\left\{\begin{array}{l}
\left(x_{1}, x_{2}, x_{3}, x_{4}, x_{5}, x_{6}\right): x_{5}+2 x_{6}= \\
0
\end{array}\right\}
$$$$
H_{9}=\left\{\left(x_{1}, x_{2}, x_{3}, x_{4}, x_{5}, x_{6}\right): x_{5}=0\right\}
$$$$
H_{10}=\left\{\left(x_{1}, x_{2}, x_{3}, x_{4}, x_{5}, x_{6}\right): x_{5}-x_{6}=0\right\}
$$$$
H_{11}=\left\{\left(x_{1}, x_{2}, x_{3}, x_{4}, x_{5}, x_{6}\right): x_{6}=0\right\}
$$$$
H_{12}=\left\{\begin{array}{l}
\left(x_{1}, x_{2}, x_{3}, x_{4}, x_{5}, x_{6}\right): x_{5}-x_{4}+ \\
x_{6}=0
\end{array}\right\}
$$$$
H_{13}=\left\{\begin{array}{l}
\left(x_{1}, x_{2}, x_{3}, x_{4}, x_{5}, x_{6}\right): x_{4}+x_{5}+ \\
x_{6}=0
\end{array}\right\}
$$

From new hypersolvable ordering we rewrite a defining polynomial as

$$
\begin{aligned}
& Q(A)=\left(x_{1}+x_{3}\right)\left(x_{1}-x_{3}\right)\left(x_{1}+x_{2}\right) x_{2} \\
& \left(x_{2}-x_{1}\right) x_{3}\left(x_{5}+3 x_{6}\right)\left(x_{5}+2 x_{6}\right) x_{5}\left(x_{5}-x_{6}\right) x_{6} \\
& \left(x_{5}-x_{4}+x_{6}\right)\left(x_{4}+x_{5}+x_{6}\right) .
\end{aligned}
$$


Note that by applying our construction we can split $A$ into two arrangements $A_{1}$ and $A_{2}$ where:

$Q\left(A_{1}\right)=\left(x_{1}+x_{3}\right)\left(x_{1}-x_{3}\right)\left(x_{1}+x_{2}\right) x_{2}$

$\left(x_{2}-x_{1}\right) x_{3}$

and

$Q\left(A_{2}\right)=\left(x_{5}+3 x_{6}\right)\left(x_{5}+2 x_{6}\right) x_{5}$

$\left(x_{5}-x_{6}\right) x_{6}\left(x_{5}-x_{4}+x_{6}\right)\left(x_{4}+x_{5}+x_{6}\right)$.

Observe that both of $A_{1}$ and $A_{2}$ are hypersolvable 3-arrangements since they have $\mathrm{Hp}$ as follows:

$\Pi^{A_{1}}=\left(\Pi_{1}, \Pi_{2}, \Pi_{3}, \Pi_{4}\right)=\left(\left\{H_{1}\right\}\right.$,

$\left.\left\{H_{2}, H_{3}\right\},\left\{H_{4}\right\},\left\{H_{5}, H_{6}\right\}\right)$

$\prod^{A_{2}}=\left(\prod_{1}, \Pi_{2}, \Pi_{3}, \Pi_{4}\right)=\left(\left\{K_{1}\right\}\right.$,

$\left.\left\{K_{2}\right\},\left\{K_{3}\right\},\left\{K_{4}\right\},\left\{K_{5}, K_{6}, K_{7}\right\}\right)$

Where;

$H_{1}=\left\{\left(x_{1}, x_{2}, x_{3}\right): x_{1}+x_{3}=0\right\}$

$H_{2}=\left\{\left(\left(x_{1}, x_{2}, x_{3}\right)\right): x_{1}-x_{3}=0\right\}$

$H_{3}=\left\{\left(x_{1}, x_{2}, x_{3}\right): x_{1}+x_{2}=0\right\}$

$H_{4}=\left\{\left(x_{1}, x_{2}, x_{3}\right): x_{2}=0\right\}$

$H_{5}=\left\{\left(x_{1}, x_{2}, x_{3}\right): x_{2}-x_{1}=0\right\}$

$H_{6}=\left\{\left(x_{1}, x_{2}, x_{3}\right): x_{3}=0\right\}$

$K_{1}=\left\{\left(x_{1}, x_{2}, x_{3}\right): x_{2}+3 x_{3}=0\right\}$

$K_{2}=\left\{\left(x_{1}, x_{2}, x_{3}\right): x_{2}+2 x_{3}=0\right\}$

$K_{3}=\left\{\left(x_{1}, x_{2}, x_{3}\right): x_{2}=0\right\}$

$K_{4}=\left\{\left(x_{1}, x_{2}, x_{3}\right): x_{2}-x_{3}=0\right\}$

$K_{5}=\left\{\left(x_{1}, x_{2}, x_{3}\right): x_{3}=0\right\}$

$K_{6}=\left\{\left(x_{1}, x_{2}, x_{3}\right): x_{2}-x_{1}+x_{3}=0\right\}$

$K_{7}=\left\{\left(x_{1}, x_{2}, x_{3}\right): x_{1}+x_{2}+x_{3}=0\right\}$

\section{(3.14) Example :}

Let $A$ be central complex 6arrangements, define as follows:
$Q(A)=x_{2} x_{1}\left(x_{1}+x_{2}\right) x_{3}\left(x_{2}-x_{3}\right) x_{4} x_{5} x_{6}$

$\left(x_{4}-x_{5}\right)\left(x_{4}+x_{5}\right)\left(x_{6}-x_{5}\right)\left(x_{6}+x_{5}\right)$

$A$ is a hypersolvable arrangement in $C^{6}$ since we can find a hypersolvable $\mathrm{Hp}$ as follows:

$\Pi^{A}=\left(\Pi_{1}, \Pi_{2}, \Pi_{3}, \Pi_{4}, \Pi_{5}, \Pi_{6}\right)=$

$\left(\left\{H_{1}\right\},\left\{H_{2}, H_{3}\right\},\left\{H_{4}, H_{5}\right\},\left\{H_{6}\right\},\left\{H_{7}\right.\right.$,

$\left.\left.H_{8}, H_{9}\right\},\left\{H_{10}, H_{11}, H_{12}\right\}\right)$ where

$H_{1}=\left\{\left(x_{1}, x_{2}, x_{3}, x_{4}, x_{5}, x_{6}\right): x_{2}=0\right\}$

$H_{2}=\left\{\left(x_{1}, x_{2}, x_{3}, x_{4}, x_{5}, x_{6}\right): x_{1}=0\right\}$

$H_{3}=\left\{\left(x_{1}, x_{2}, x_{3}, x_{4}, x_{5}, x_{6}\right): x_{1}+x_{2}=0\right\}$

$H_{4}=\left\{\left(x_{1}, x_{2}, x_{3}, x_{4}, x_{5}, x_{6}\right): x_{3}=0\right\}$

$H_{5}=\left\{\left(x_{1}, x_{2}, x_{3}, x_{4}, x_{5}, x_{6}\right): x_{2}-x_{3}=0\right\}$

$H_{6}=\left\{\left(x_{1}, x_{2}, x_{3}, x_{4}, x_{5}, x_{6}\right): x_{5}=0\right\}$

$H_{7}=\left\{\left(x_{1}, x_{2}, x_{3}, x_{4}, x_{5}, x_{6}\right): x_{4}=0\right\}$

$H_{8}=\left\{\left(x_{1}, x_{2}, x_{3}, x_{4}, x_{5}, x_{6}\right): x_{6}=0\right\}$

$H_{9}=\left\{\left(x_{1}, x_{2}, x_{3}, x_{4}, x_{5}, x_{6}\right): x_{4}-x_{5}=0\right\}$

$H_{10}=\left\{\left(x_{1}, x_{2}, x_{3}, x_{4}, x_{5}, x_{6}\right): x_{4}+x_{5}=0\right\}$

$H_{11}=\left\{\left(x_{1}, x_{2}, x_{3}, x_{4}, x_{5}, x_{6}\right): x_{6}-x_{5}=0\right\}$

$H_{12}=\left\{\left(x_{1}, x_{2}, x_{3}, x_{4}, x_{5}, x_{6}\right): x_{6}+x_{5}=0\right\}$

Note that $A$ is supersolvable arrangement since

$\ell(A)=r k(A)=6$

From new hypersolavble ordering we rewrite the defining polynomial of $A$ as follow:

$$
\begin{aligned}
& Q(A)=x_{2} x_{1}\left(x_{1}+x_{2}\right) x_{3}\left(x_{2}-x_{3}\right) x_{4} \\
& x_{5}\left(x_{4}-x_{5}\right)\left(x_{4}+x_{5}\right) x_{6}\left(x_{6}-x_{5}\right)\left(x_{6}+x_{5}\right)
\end{aligned}
$$


Mayadah .A

Note that by applying our construction we can split

$A$ into two 3-arrangements $A_{1}$ and $A_{2}$ where:

$Q\left(A_{1}\right)=x_{2} x_{1}\left(x_{1}+x_{2}\right) x_{3}\left(x_{2}-x_{3}\right)$ and

$Q\left(A_{2}\right)=x_{2} x_{1}\left(x_{1}-x_{2}\right)\left(x_{1}+x_{2}\right) x_{3}$

$\left(x_{3}-x_{2}\right)\left(x_{3}+x_{2}\right)$

Observe that both of $A_{1}$ and $A_{2}$ are supersolvable arrangements since they have Hp as follows:

$\prod^{A_{1}}=\left(\prod_{1}, \prod_{2}, \prod_{3}\right)=\left(\left\{H_{1}\right\},\left\{H_{2}, H_{3}\right\}\right.$,

$\left.\left\{H_{4}, H_{5}\right\}\right)$

$\prod^{A_{2}}=\left(\prod_{1}, \prod_{2}, \prod_{3}, \Pi_{4}\right)=\left(\left\{K_{1}\right\},\left\{K_{2}\right.\right.$, $\left.\left.K_{3}, K_{4}\right\},\left\{K_{5}, K_{6}, K_{7}\right\}\right)$

\section{References:}

[1] Arnold, V. I., The Cohomology Ring of The Colored Braid Group. Mat. Zametki 5 (1969) 227-231.

[2] Brieskorn, E., Sur les Groupes de Tresses. Séminaire Bourbaki 1971/72. Lecture Notes in Math. 317, Springer, 1973, pp. 21-44.

[3] Jambu, M. and Papadima, S., $A$ Generalization of Fiber type Arrangements and a new Deformation Method. Topology, (1998) 3, pp. 1135-1164.

[4] Jambu, M. and Papadima, S., Deformation of Hypersolvable Arrangements. Topology and it is Applications (2002) pp. 103-111.

[5] Ali, H. M., A Topological Interpretation for Vanishing of Higher Homotopy Groups of a Hypersolvable Arrangement. .Ph.D thesis of University of Basra in (2007)
[6] Al-Ta'ai, A.H.,Ali, H.M., Majeed, M.A., On Orlik-Solomon algebra of a hypersolvable Arrangement, Basra Researchers J.36(2010).19-43.

[7] Mahdi, R. S., Product of Hypersolvable Arrangements. Ph.D thesis of University of Basra in (2006).

[8] Orlik, P. and Solomon, L., Combinatorics and Topology of complements of hyperplanes, Invent. Math. 56 (1980), pp. 167-189.

[9] Orlik, P. and Terao, H., Arrangements of Hyperplanes, Grundlhern Math. Wiss., vol. 300, Springer-Verlag Berlin, (1992).

[10] Rota, G. C., On the Foundations of Combinatorial Theory I. Theory of the Möbius Functions, Z. Wahrsheinlichkeitstheorie 2, (1964), pp. 340368.

[11] Sagan, B. E., A Generalization of Rota's NBC Theorem, AMS subject classification, (1991).

[12] Stanley, R. p., Supersolvable Lattices. Algebra Universals 2, (1972), pp. 197-217.

[13] Falk, M. and Terao, H., $\beta$ NBC-Bases for Cohomology of Local Systems on Hyperplane Complements, available at arxiv:alg-geom / 9412009 v3, (24-May1995). 


\section{(حول قواعد-NBC لضرب الترتيبة القابلة للحل الفوقية)

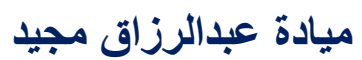 جامعة البصرة /كلية العلوم/رياضيات}

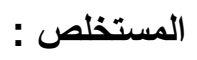

الهدف من هذا البحث يتمركز حول دراسه ضرب الترتيبه القابله للحل فوقيا التي تم دراستها باستخدام مفهوم تجزئه الترتيبه القابله للحل فوقيا ففي هذا البحث تمكنا من برهان انه اذا كان A×B ترتيبه قابله للحل فوقيا فأن كل من

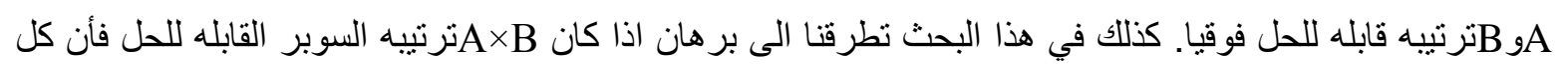
من B تكون ترتيبه سوبر قابله للحل وايضا كيفية برهان ان بعد اول زمرة غير متلاشية الاعلى هوموتوبي لمتممة . $\mathrm{p}(\mathrm{A} \times \mathrm{B})=\min \{\mathrm{p}(\mathrm{A}), \mathrm{p}(\mathrm{B})\}$ تكون M(A×B) 